Article

\title{
Enabling Multiple Access in Visible Light Communication Using Liquid Crystal Displays: A Proof-of-Concept Study
}

\author{
MD Rashed Rahman ${ }^{(D)}$ Kehinde Adedara and Ashwin Ashok *(D) \\ Department of Computer Science, Georgia State University, Atlanta, GA 30303, USA; \\ mrahman19@student.gsu.edu (M.R.R.); kadedara1@student.gsu.edu (K.A.) \\ * Correspondence: aashok@gsu.edu
}

Received: 19 April 2020; Accepted: 13 May 2020; Published: 16 May 2020

check for updates

\begin{abstract}
The directionality of optical signals provides an opportunity for efficient space reuse of optical links in visible light communication (VLC). Space reuse in VLC can enable multiple-access communication from multiple light emitting transmitters. Traditional VLC system design using photo-receptors requires at least one receiving photodetector element for each light emitter, thus constraining VLC to always require a light-emitter to light-receptor element pair. In this paper, we propose, design and evaluate a novel architecture for VLC that can enable multiple-access reception using a photoreceptor receiver that uses only a single photodiode. The novel design includes a liquid-crystal-display (LCD) based shutter system that can be automated to control and enable selective reception of light beams from multiple transmitters. We evaluate the feasibility of multiple access on a single photodiode from two light emitting diode (LED) transmitters and the performance of the communication link using bit-error-rate (BER) and packet-error-rate (PER) metrics. Our experiment and trace based evaluation through proof-of-concept implementation reveals the feasibility of multiple LED reception on a single photodiode. We further evaluate the system in controlled mobile settings to verify the adaptability of the receiver when the LED transmitter changes position.
\end{abstract}

Keywords: visible light communication; multiple access communication; high data rate; spatial multiplexing; mobile VLC

\section{Introduction}

Visible Light Communication (VLC), is an emerging wireless communication technology that operates unregulated in the visible-light band (400-800 THz frequencies or 380-780 nm wavelengths) of the electromagnetic spectrum, and is enabled by light emitting elements such as light emitting diodes (LED) and light receiving elements such as photodiodes (PD). Due to directionality of light beams, VLC is a line-of-sight (LOS) technology that requires the light transmitter and receiver to be within each others field-of-view (FOV) [1]. The LOS requirement provides novel opportunities for efficient space and time reuse in VLC where multiple light emitting transmissions could be multiplexed.

Traditional VLC [2] that operates using a single non-array photodiode receiver based reception, requires to incorporate specific multiple access mechanisms to enable reception from different light emitters. By leveraging the directionality of optical signals and that light emitters can be spatially differentiated, it is possible to multiplex signals by combining space division multiple access (SDMA) with time/frequency/code (TDMA/FDMA/CDMA) division access schemes, however, the nature of photoreceptors to collectively add all the detected photons within its FOV limits makes differentiation of multiple transmissions and from ambient noise very challenging. This limits VLC to the effective 
communication using only one light beam (or transmitter) at each instance of time. The key challenge in using multiple access mechanisms in single-photodiode non-array VLC receiver systems is that the incoming signals, through may be spatially and temporally separated, but once they reach the receiver collector (lens), the signals are mixed (leading to interference) with each other and thus cannot be differentiated. Unlike radio-frequency communication, where polarity of signals and thus representing signals as complex numbers is possible, in optical wireless, the received signals are essentially the positive-sum of all photons, which carry no polarity. With stringent constraints that there cannot be an extra channel and that the signals must be easily identified from each LED on the photodiode, multiple access in VLC is very challenging without the use of array receivers or side information. Therefore, unless there is extra information regarding the signals (possibly through an extra control channel) or custom detection mechanisms incorporated into the system, the ability to spatially and temporally differentiate optical signals using a single non-array photodiode receiver remains an open challenge.

Multiple-Input Multiple-Output (MIMO) architectures for VLC that have been proposed and designed before [3,4] require multiple photoreceptor elements, for example, as in photodetector arrays and image sensing arrays or cameras. The use of array elements in a receiver limits the sampling bandwidth (photodiode sampling frequency or camera frames-per-second) of the receiver hence limiting the achievable throughput of the system.Therefore, enabling multiple-access while retaining the high-speed sampling capacity of photodiodes is the other key open challenge that remains to be addressed.

To address the challenges presented above, this paper explores the use of spatial filtering mechanisms using a new hardware design for VLC receivers, to enable multiple access. In essence, this work presents a proof-of-concept study of using liquid crystal displays (LCD) to potentially enable multiple access in single non-array photodiode receivers. We propose a receiver design that uses LCDs to differentiate multiple LED transmissions and enable multiplexed communication using a single photodiode in the receiver. To this effect, we build our system over a baseline architecture from our prior work [5], where a liquid crystal display (LCD) panel was used as a digital gate or shutter, to allow or disallow signals onto a photodiode receiver. In this paper, we extended this baseline design to build a single photodiode receiver equipped with a LCD shutter array controlled by an automated signal selection protocol to differentiate reception from multiple LED transmissions (conceptual diagram in Figure 1). This protocol helps relax the assumption (considered in our prior work) of the apriori knowledge of which LCD shutter should be opened (or closed), and enables on-the-fly determination of the intended shutter state. The proposed receiver architecture, to be referred to as pixelated shutter receiver for the rest of the paper, sets the foundation for future VLC system architectures to achieve MIMO communication using only a single photodiode receiver. Achieving MIMO first requires multiple access reception capability demonstration, and to be best of our knowledge, our work in this paper presents the first design and evaluation of a novel multiple access VLC receiver. In summary, the key contributions of this paper are:

1. Design of an automated shutter control protocol for selective reception of multiple LEDs using a pixelated shutter receiver.

2. Implementation of a prototype pixelated shutter receiver multiple access system with $2 \times 2 \mathrm{LCD}$ panels, single photodiode and a software-defined radio.

3. Experimental trace based evaluation of the pixelated shutter receiver which employs the bespoke proposed automated shutter protocol, for 2 independent LED transmitter scenario using (a) bit-error, (b) packet error and (c) latency metrics.

4. Experimental evaluation of the automated shutter control protocol under controlled mobile environment. 


\section{Related Work}

Background. In our prior work [5], we introduced a new architecture for VLC that uses a high-speed photodetector and an LCD shutter acting as a programmable image sensor aperture. In this work, our measurement studies proved that noise and interference can be separated spatially using our VLC receiver to improve the Signal to Noise Ratio (SNR) and Signal to Noise Interference Ratio (SINR) significantly. Through a proof-of-concept experimentation, in our previous work [5] we have studied the feasibility of noise and interference reduction by manually selecting one of the shutter pixel apertures for higher signal reception. In this paper, we relax the assumption (considered in our prior work) of the a priori knowledge of which shutter should be opened (or closed), and advance the design by proposing a novel automated shutter control to help differentiate LED signals on the photodiode. This paper leads and consolidates the idea of multiple access in VLC by adopting a shutter controlling algorithm in the receiver. We evaluate the system for high speed reception (up to $2 \mathrm{MHz}$ data transmission frequency) for multiple access from 2 LEDs and feasibility of automation under controlled mobile settings.

In the rest of this section, we discuss some of the existing works that are closely related to the challenges targeted in our system. However, we emphasize that no prior work has shown the capability of achieving high-speed multiple access in VLC using a single photodiode receiver, which remains the key focus of this paper's contribution.

Existing VLC technologies. Recent years have seen active development of VLC research prototype systems. However, these systems have specific limitations owing to the customizations in their hardware/software design. PureLiFi [6] devices have shown the capability of Mbps-Gbps data rates, but their performance is limited to static VLC settings and carry high hardware overheads, complex signal processing, thus requiring a huge cost for the product. OpenVLC1.3 [7] is an open-source embedded VLC prototype based on simpler protocols that offers data rates up to $400 \mathrm{kbps}$. The customized hardware and requirement of modifying the operating system kernel makes this option very challenging to generalize. With a $1.4 \mathrm{kbps}$ data rate, LocalVLC [8] presents a low-cost VLC prototype for indoor IoT applications based on morse code modulation, however, fails to motivate its usage for typical indoor IoT applications where higher throughput is typically required. Other VLC systems including Purple VLC [9-11], have similar challenges as discussed above. With achieving high data rate remaining a key challenge for existing VLC systems, the addition of multiple access requirement can either require significant modifications to these designs or may not be feasible at all. Our proposed design can potentially address high-data rate, multiple access and mobility all at once through a unified design. By using the shutter control mechanism efficiently, signals can be differentiated from noise and interference, thus enabling cleaner signal reception or higher Signal-to-Noise (SNR) and/or Signal-to-Interference-and-Noise Ratios (SINR). With a cleaner signal, the signal modulation mechanisms can be kept simple with more focus laid on achieving multiple access, high-throughput reception and mobility.

Non-Orthogonal Multiple Access in VLC. Recent works have proposed non-orthogonal multiple access (NOMA) schemes for VLC [12-14] to improve spectral efficiency and enable multiple access in VLC. However, the common challenge in these designs is the reliance on channel state information (CSI) and the requirement for the transmitter and receiver to be informed a priori about CSI. For example, the work in Reference [12] introduces a power domain based multiple access protocol so that the users can use the entire bandwidth during the communication session, but requires the CSI and only works in small indoor environments. NOMA is a good contender for multiple access in VLC however the designs have been largely limited to showing the feasibility of interference cancellation under strong assumptions which limit the effective throughput performance of the VLC system.

Multiple Access using MIMO Techniques. Using the MIMO technique proposed in Ref. [15], several works have presented different multiple access schemes using different equalization [16] and modulation schemes such as OFDM [17,18], optical spatial modulation (OSM) with OFDM [19]. Reference [20], introduces an Optical Code Division Multiple Access (OCDMA) technique and 
Reference [21] used intensity modulation to support multiple users in MIMO VLC system. A key challenge with photodiode arrays is that they allow more noise, from ambient light (sunlight and artificial lighting), into the receiver due to the wide field-of-view, thus affecting received signal quality and data rate. The work in Reference [22] proposes multi-color LEDs based MIMO VLC system to ensure higher data rates (upto $1 \mathrm{Gbps}$ ) but the system requires complex signal processing and equalization techniques. Reference [23] presents a precoding technique to mitigate inter-cell and intra-cell ambient light interferences in multi-cell VLC systems to improve the bandwidth efficiency, where specific spatial regions are considered as cells, similar to cellular communication. The complexity of the MIMO techniques for real-time implementation and performance, and the necessity of high efficiency and costly photoreceptors (avalanche photodiodes [4]) for improving data rates, remain challenges yet to be solved for MIMO VLC systems.

Wavelength Division Multiplexing (WDM). In Reference [24], the authors introduce a bi-directional VLC in full duplex mode by parallel transmission of three (RGB) channels and use OFDM modulation demodulation to increase the aggregate data rate. In another work [25], a color-shift keying CDMA (CSK-CDMA) based VLC system has been developed to increase the VLC throughput and for allowing multiple access.To provide ultra high data rates (>35 Gb/s) in a wide range of coverage, a WDM system of four-colour multiplexed using MEMS based beam-steering has been presented by Chun et al. [26]. While such systems could potentially avoid interference across specific wavelengths, the complexity, high bit-error-rates, and costly hardware elements limit the usage of these approaches.

\section{System Architecture}

We have designed a pixelated shutter based VLC receiver that automatically identifies and selects/isolates signals from multiple LEDs. The key components of the system, as illustrated in Figure 1 (left), include a photodiode (capable of high-speed sampling), a LCD shutter array, a shutter control unit, a computing unit, and a condenser lens for optical focusing. The key idea of proposed design is to allow signals from multiple LED transmitters to be correctly detected and decoded using a single photodetector VLC receiver. With the knowledge and assurance that only one LED signal impinges on the photodiode at each of its sampling instance, the receiver can be operated at the bandwidth matched to the LED's transmission, resulting in high SNR and thus potentially high data rate reception.
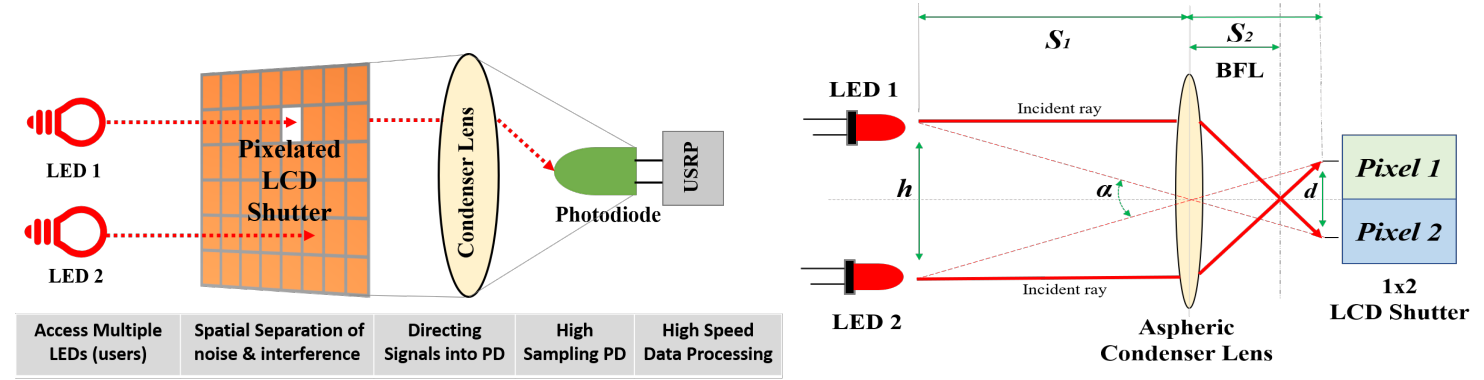

Figure 1. (left) Conceptual diagram of pixelated shutter visible light communication (VLC) receiver. (right) LED Positioning geometrical analysis to ensure focus of only 1 LED per shutter pixel.

The shutter uses LCDs which act as a digital aperture that allows (disallows) the impinging light beams, to reach the photodiode, based on the input voltage to the shutter. Using this digital aperture as a control the receiver is able to select which of the incoming light beams are to be decoded by the photodiode at each instance of time. The computing unit at the receiver hosts the decoding algorithms and mechanisms to efficiently decode the signal that has been selected. The digital control of the shutter is integrated with the decoding modules in the computing unit, such that there is active feedback on the quality of the received signal. The feedback information includes the received signal-to-noise-ratio (SNR) and a digital identification of the signal using packet header bits. This design enables a seamless 
functioning of the selective control of the reception and the decoding in tandem. The selection of the desired signal(s) is a one-time process and needs to be repeated only when there is mobility or during link failures.

\subsection{Spatial Multiplexing Using Pixelated Shutter}

We describe the multiple access capability through the spatial multiplexing setup illustrated in Figure 1 (right). Consider two LEDs placed in space (at same height but separated along the horizontal) aiming to focus their signals (using the condenser lens) onto a photodetector (not shown in Figure 1 (right)) by passing through a $1 \times 2$ LCD shutter pixel system, where the pixels are aligned next to each other along the horizontal axis. Let us consider that each pixel $i$ is responsible for signals from corresponding LED $i$. In this way, when the signals from the LEDs are beamed onto the photodiode, each pixel can selectively allow/disallow the signals provided the signals are independently identified (and differentiated) and the information on which signal (LED) should be selected is feedback to the shutter control unit (not shown in Figure 1 (right)).

To ensure that the signals do not overlap onto a single shutter surface area, our design requires that each LED signal can be spatially separated onto independent shutter pixels. This depends on the size of the shutter, distance of communication and the spatial separation of the LEDs. Through lens equation [27] and using simple trigonometrical calculations, we derive that the minimum distance of separation between the LEDs must be $h=d S_{1} / B F L$ where $d$ is the distance between two shutter pixel centers (considering a square pixel it is the pixel side length), $S_{1}$ is the distance from LED to the lens, and BFL is the back focal length which is the focal length of the lens. We also derive the minimum angle of separation between the LED beams as $\alpha=2 \arctan \left(\frac{h}{2 S_{1}}\right)$. These equations provide the designer the control of placing the LEDs in space so as to allow multiple access reception on the single photodiode receiver. We determine that the minimum horizontal separation between the LEDs has to be $h=14.88 \mathrm{~cm}$ and $\alpha=51.2^{\circ}$ for the component values from our proof-of-concept prototype system (section 4), where $S_{1}=15.5 \mathrm{~cm}, S_{2}=8.2 \mathrm{~cm}$ and $B F L=3.75 \mathrm{~cm}$ and $d=3.6 \mathrm{~cm}$, radiation angle of the LED is $50^{\circ}$, at a distance of $10 \mathrm{~m}$ the equivalent minimum separation distance would be close to $10 \mathrm{~m}$, however, this distance can be reduced if the $d$ were to be increased. By merely doubling the size $(d)$ of the shutter, the required distance $h$ now can be $5 \mathrm{~m}$. This means that the selection of separation between LEDs in space and the size of the shutter involves a tradeoff. The tradeoff between $h$ and $d$ can also be adjusted by using LASER type emitters or LEDs which have smaller radiation angle.

\subsection{Automated Shutter Control Protocol Design}

The shutter control protocol operates in tandem with the decoding process running in the computing unit connected to the photodiode. The control protocol, discussed in Algorithm 1 and demonstrated as block diagram in Figure 2 involves two steps:

Step 1: Discovery phase, where the receiver does a preliminary pruning of all signals that do not represent a transmit signal by using the signal-to-noise-ratio from each shutter pixel $i\left(S N R_{p x i}\right)$. SNR is computed as ratio of signal power to noise power, where power is computed as the mean-squared photodiode voltage reading divided by the sampling time interval. This step helps to filter ambient light, DC noise sources and other known noise sources. This way, only a subset of the shutter pixel array are kept OPEN and are to be processed, thus limiting the processing to a smaller subset of signals.

Step 2: Identification phase, where the receiver does a fine tuning of identifying each transmit signal and selectively opening the corresponding shutter pixels to allow/disallow the signal for continued reception at the photodiode. The identity of the signals are maintained through unique header sequences (barker codes) in the data packets. We consider that a unique ID of each transmitter will be registered at the receiver apriori during the first setup of the system (one time) and update the identity look-up table as necessary. 


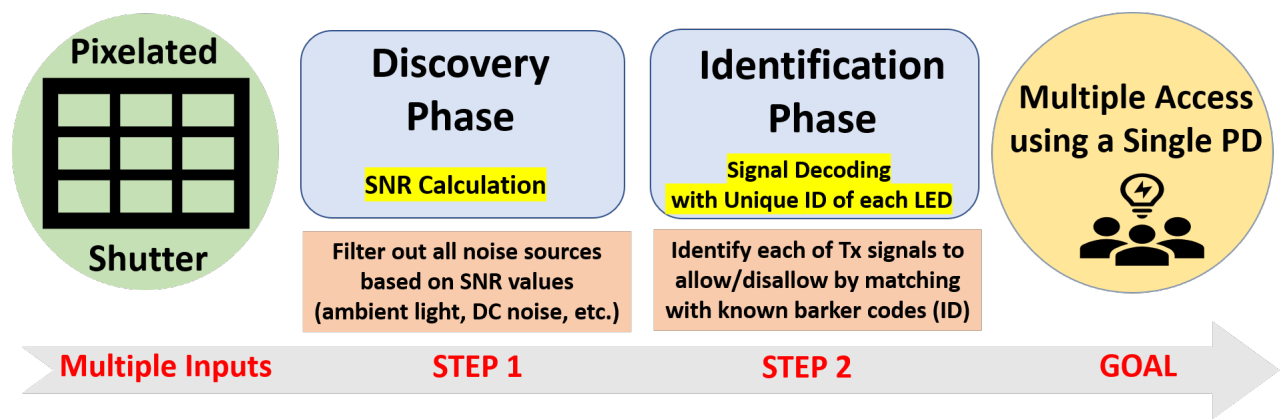

Figure 2. Block Diagram of Shutter Controlling Algorithm.

The shutter control protocol enables multiple access where information from multiple LED transmitters can be decoded by a single photodiode receiver. By enabling which signals to receive at which instance of time, the receiver can choose time-slots to receive and decode specific signals. Our system by default functions as a space and time-division multiple access (SDMA and TDMA) system where each transmission is decoded across a time-slot duration of $T_{S}$ seconds, and the selection of spatially separated LED emissions is controlled through the LCD functions. While transmitters could potentially transmit at different rates and that time slots of transmission and reception may incur synchronization issues, the spatial separation through the LCD enables to first find which signal is intended and which is not, and then physically allow only the signal intended. Without the LCD the other possibility to achieve the same functionality is to use an array receiver, however, as mentioned before, array receivers can significantly increase the complexity of the system. This work aims to study how a non-array receiver could still function such multiple access schemes, albeit with some practical and minimalist hardware additions.

The choice of the value of $T_{S}$ depends on the application. For example, a slot duration of 1-2 s may work for beaconing and repetitive transmission such as in sensor or IoT applications, however, for streaming applications the slot has to be made much smaller (order of few ms). A smaller slot duration also implies that the pixel switching control must happen as fast as the selected slot duration. Depending on the type of LCD shutters, the switching time can vary from few micro to $10 \mathrm{~s}$ of milli seconds.

\section{Prototype Implementation}

We implemented a prototype pixelated shutter receiver as shown in the setup in Figure 3. The design parameters are all listed in Table 1 . The key components of the hardware system include 2 RED LEDs, a PDA10A2 Amplified Photodetector, a custom made $1 \times 2$ pixelated LCD shutter and an aspheric condenser lens (outer diameter $80 \mathrm{~mm}$ and $\mathrm{BFL}=37.5 \mathrm{~mm}$ ). We implemented the automated shutter control algorithm in a Raspberry Pi 3 Model B+ which interfaces with a $2 \times 2$ pixelated shutter, built using off-the-shelf LCD shutter elements from AdafruitDue to the leakage of LED light we use the $2 \times 2$ LCD setup in $1 \times 2$ reception mode. We used two N210 USRPs as the computing units at the transmitter (controlling LED transmissions) and receiver ends (decoding signals from photodiode). We used a LFTX daughterboard capable of operation from $0-30 \mathrm{MHz}$ and a RFTX daughterboard. The 2 LEDs were controlled using two different USRPs, each hosting a LFTX, and one of the USRPs hosting a LFRX that also conducted the reception. We used GNU Radio blocks (block diagram of GNU applications shown in Figure 4) to transmit and receive signals using the USRPs. We chose to use the state-of-the-art Gaussian Minimum Shift Keying (GMSK) as the modulation strategy in our design, however, any type of modulation can be used in the system. We use 13-bit and 11-bit barker sequences for LED1 and LED2 header bits, respectively. We have implemented the transmissions in the form of UDP packets of size 2096 bits (as per IEEE 802.15.7 VLC standard [28] packet definitions). 

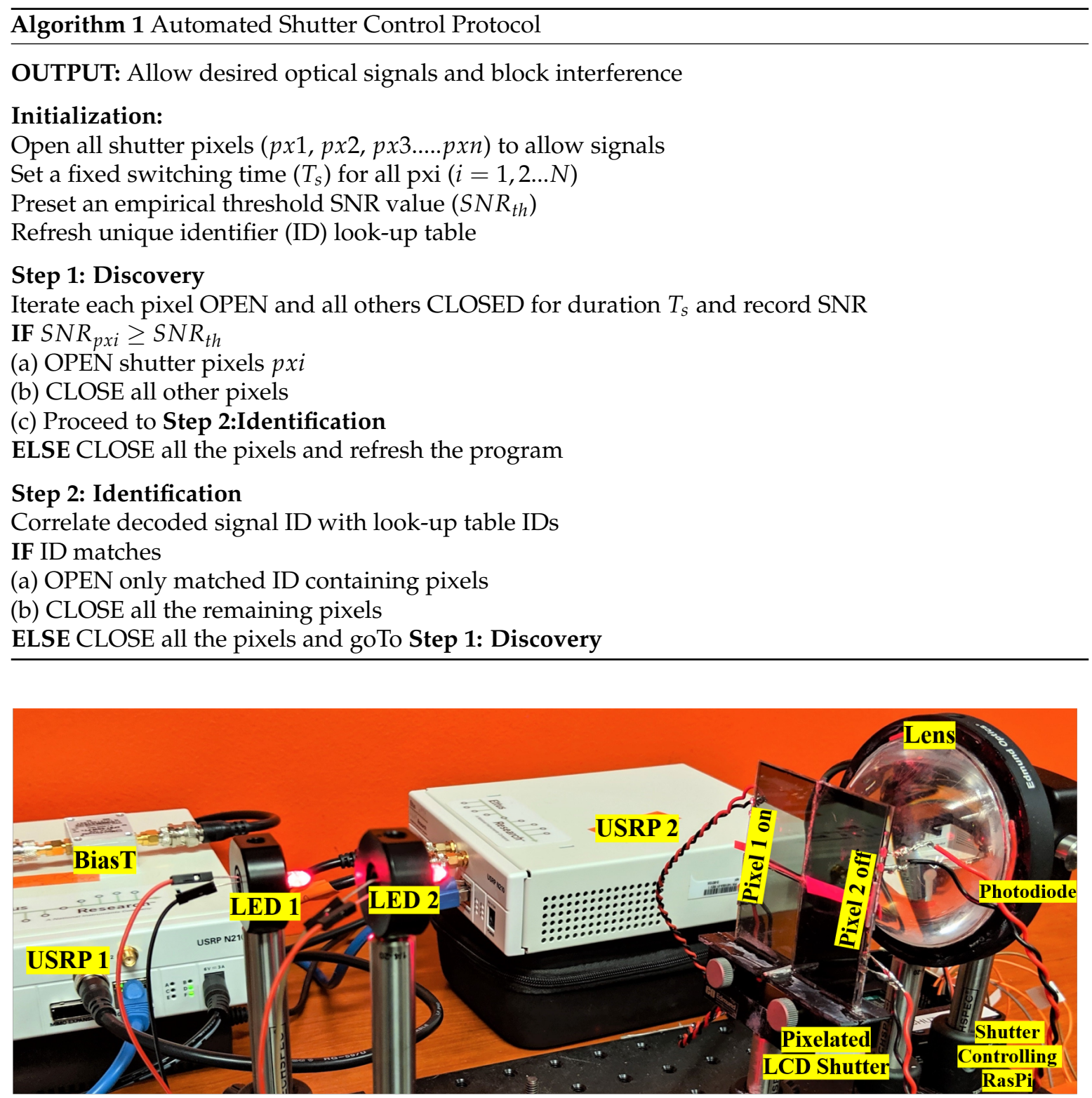

Figure 3. General setup of the pixelated shutter receiver system. This picture shows a 2 LED transmitter setup with a single photodiode receiver and $2 \times 2$ liquid-crystal-displays (LCDs) fit in a $1 \times 2$ shutter pixels configuration. 


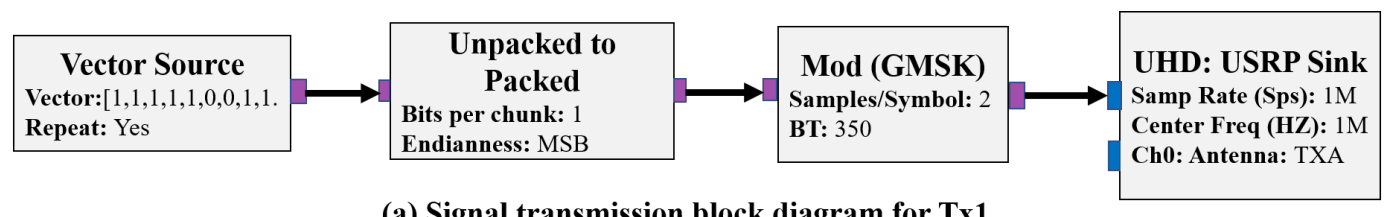

(a) Signal transmission block diagram for Tx1

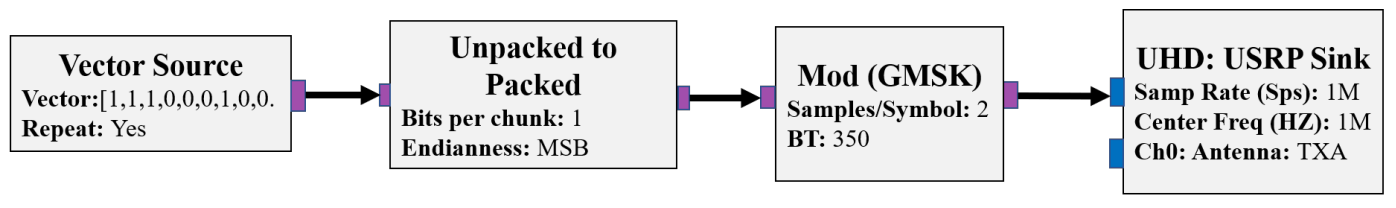

(b) Signal transmission block diagram for $\mathrm{Tx} 2$

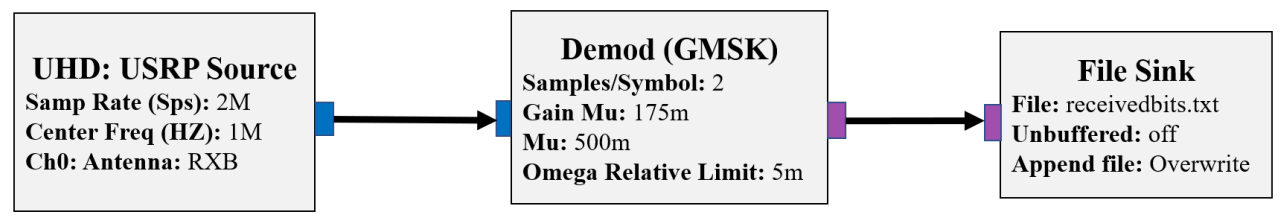

(c) Signal reception block diagram for single $\mathbf{R x}$

Figure 4. USRP GNU Radio Block Diagram for (a) transmitter LED 1, (b) transmitter LED 2, a and (c) pixelated shutter receiver. Note that the vector source values shown are only example values. The setup uses 2 N210 USRPs with LFTX daughterboard for the transmitters and 0-30 MHz LFRX daughterboards for the receiver.

Table 1. LED, Photodiode, Lens and LCD specifications.

\begin{tabular}{|c|c|}
\hline \multicolumn{2}{|c|}{ LED Specifications } \\
\hline Number of LEDs & 02 \\
\hline LED Type and Size & Red, T-1 3/4 (5 mm) \\
\hline Horizontal distance between LED's [cm] & $14.88 \mathrm{~cm}$ \\
\hline Viewing Angle of LED [deg.] & $30^{\circ}$ \\
\hline Luminous Intensity at each LED [mcd] & $3500 \mathrm{mcd}$ \\
\hline Optical Output Power at each LED [mW] & $125 \mathrm{~mW}$ \\
\hline Wavelength of each LED [nm] & $650 \mathrm{~nm}$ \\
\hline \multicolumn{2}{|c|}{ Photodiode (PD) Specifications } \\
\hline Physical Active Area of the PD $\left[\mathrm{mm}^{2}\right]$ & $0.8 \mathrm{~mm}^{2}$ \\
\hline Wavelength Range of PD [nm] & 200 to $1100 \mathrm{~nm}$ \\
\hline Bandwidth of PD [MHz] & $150 \mathrm{MHz}$ \\
\hline Peak Response of PD [A/W] & $0.44 \mathrm{~A} / \mathrm{W}$ \\
\hline \multicolumn{2}{|c|}{ Lens Specifications } \\
\hline Lens Type & Aspheric Condenser Lens \\
\hline Outer Diameter [mm] & $80 \mathrm{~mm}$ \\
\hline Back Focal [mm] & $37.5 \mathrm{~mm}$ \\
\hline \multicolumn{2}{|c|}{ LCD Shutter Specifications } \\
\hline LCD Type & TN, Transmissive, Positive \\
\hline Dimensions of LCD Pixel & $36 \times 36 \mathrm{~mm}$ \\
\hline Driving Voltage of LCD [V] & $3-5 \mathrm{~V}$ \\
\hline Maximum Opaqueness (\%) & $95 \%$ \\
\hline
\end{tabular}

\section{Evaluation}

We evaluate our system to study the feasibility of our system to achieve high speed reception and the performance of the automated shutter protocol for multi channel visible light signal reception on a single photodiode receiver. All the experiments were conducted in a lab setting, indoors, under ceiling 
white ambient lighting. Unless mentioned, the distance between the LED and photodiode in our experiments was set to $15.5 \mathrm{~cm}$.

\subsection{BER Analysis}

We conducted two types of experiments to evaluate the bit error rate performance of our system: (a) BER under different types of interference, and (b) BER under selective mapping of signal and shutter pixels. We calculated the BER as the ratio of total number of bit decoding errors to the total number of transmitted bits per trial. The BER experiments involved, in general, transmitting a random stream of 30,000 bits and logging the decoded bits at the receiver. Each experiment trial was repeated 5 times, BER was computed per trial and the average BER is reported. Unless otherwise specified, the BER values reported in this paper refer to the average BER over 5 experiment trials. We chose LED 1 as the desired transmitter and LED 2 as interference. The LEDs were modulated using the baseband signal from the USRP, where a pulse waveform at a specific (generation) transmit frequency was input to the LEDs which mapped a 1 to pulse HIGH and 0 to pulse LOW. Since the operation was in baseband, the transmit frequency is essentially equal to the transmitter data rate. The data rates were thus chosen as per the experiment goals:

- Goal A: The 100 bits/sec is chosen arbitrarily, as the primarily goal of this experiment is to validate the additive nature of optical signal at the photodiode receiver.

- Goal B: We chose operation at (500 k, 1 M, 2 M) bits/sec rates and measured the BER and PER at different shutter configurations at those rates for our proposed pixelated LCD architecture. The $2 \mathrm{Mbps}(2 \mathrm{MHz})$ limit for evaluation was due to the limited operable range of the LFTX/LFRX USRP daughterboard. In our future work we intend to evaluate our system for Gbps range using SLD Laser type transmitters and using FPGA (Field-programmable Gate Array) computing nodes.

\subsubsection{BER and Interference Patterns (Goal A)}

We set the transmit frequency to be $100 \mathrm{~Hz}$ and conducted the BER experiment under four signaling types:

- Type 1: Only the transmit signal.

- Type 2: The transmit signal and ambient DC noise.

- Type 3: Transmit signal and Interference signal sending identical patterns in phase.

- Type 4: Transmit signal and Interference signal sending identical patterns at 180 deg out-of-phase.

We report the BER from these experiments in Table 2. We can observe from the BER values from Case 1 that the error rates for the system are generally high when the desired signals and the interference are combined at the photodetector. We observe that the BER is practically low (for feasible data communication) when the pixels are selectively OPEN/CLOSE to allow only the desired signal, which has been achieved without major changes to the receiver. We can also observe from the BER values, the additive property of the receiver, where the BER is low when the interference signal is identical and of same phase (as the effective received signal amplitude is doubled) and high when the same interfering signal is out-of-phase.

Table 2. Bit error rate (BER) at $100 \mathrm{~Hz}$ signaling under different interference patterns. Case 1: All pixels OPEN, Case 2: Only desired signal pixel is OPEN.

\begin{tabular}{llllllll}
\hline \multicolumn{2}{c}{ Type 1 } & \multicolumn{2}{c}{ Type 2 } & \multicolumn{2}{c}{ Type 3 } & \multicolumn{2}{c}{ Type 4 } \\
\hline Case I & Case II & Case I & Case II & Case I & Case II & Case I & Case II \\
\hline $5 \times 10^{-4}$ & $5 \times 10^{-4}$ & $4.9 \times 10^{-1}$ & $8 \times 10^{-4}$ & $2.2 \times 10^{-4}$ & $2.1 \times 10^{-4}$ & $4.9 \times 10^{-1}$ & $5.1 \times 10^{-4}$ \\
\hline
\end{tabular}




\subsubsection{BER and Selective Signaling (Goal B)}

Consider pixel-1 as the pixel corresponding to LED 1 and pixel-2 as the one for LED 2. We conducted the BER experiments under three different configurations of the shutter pixel and under three different transmit frequencies. The results from Table 3 indicate that the BER is at least an order low when only the desired signal is received versus when the interference is also sampled on the single photodiode receiver. The BER values, though relatively high (which can be reduced using error control coding) for data streaming applications, however, indicate the feasibility of multiple access using our proposed architecture. Considering Goodput $\approx(1-B E R) *$ transmitsymbols $/ \mathrm{sec} *$ bits / symbol $*$ errorcontrolcoderate, we note with an assumed code rate of $1 / 2,2 \mathrm{Mhz}(2 \mathrm{M}$ symbols/sec) transmit rate and 2 bits/symbol modulation rate, the effective Goodput per LED in our preliminary system is about 1.9 Mbps.

Table 3. BER at different shutter pixel configurations. Configuration 1: ONLY pixel-1 is OPEN. Configuration 2: ONLY pixel-2 is OPEN. Configuration 3: BOTH, pixel-1 and pixel-2 are OPEN.

\begin{tabular}{cccc}
\hline Frequency (Hz) & Configuration $\mathbf{1}$ & Configuration 2 & Configuration 3 \\
\hline $500 \mathrm{kHz}$ & 0.015 & 0.039 & 0.21 \\
$1 \mathrm{MHz}$ & 0.015 & 0.035 & 0.21 \\
$2 \mathrm{MHz}$ & 0.015 & 0.030 & 0.21 \\
\hline
\end{tabular}

We observed after our experiments and analysis that the positioning of our custom built LCD array on pixel 2 location was slightly tilted thus causing a focussing issue of any light beam falling on it to the photodiode. The lens was placed at exactly the optical focal length distance from the photodiode to ensure convergence of the rays, however, due to the tilt the LED 2 signal falling on pixel 2 was actually defocused. After a breakdown of the equipment we measured that the signal intensity was reduced by almost $50 \%$. This actually confirms our finding that the BER is little more than $2 \times$ that of LED1-pixel 1. We report these numbers as is and believe it is a honest representation of our experiments and that it actually helps make key observations.

\subsection{PER and Signal Selection}

We conducted 5 PER evaluation trials each for considering LED 1 or LED 2 as the desired signal. We collected traces from these trials and determined the PER through offline calculations. In each trial we transmit a continuous stream of packets of size 2096 bits, where each packet has a random stream of bits as payload and a 13 bits header. The header served as the unique ID for each LED. The header would be the same for all packets from a specific transmitter. For LED 1 we chose a 13 bits sampled from barker sequences as ID, and for LED 2 we chose a 11 bits sampled from barker sequence plus a 2 bit (11) padding. We chose the time slot $\left(T_{s}\right)$ duration to be 2 seconds for each iteration over a pixel. We recorded the received and decoded bits from packets in each iteration of pixel OPEN cycles. We collected the received signal traces from each iteration of each STEP of the automated shutter control protocol (Algorithm 1). We iterated over 1 cycle of each pixel OPENING and then choosing either of the pixels that corresponds to the desired signal. We can observe from Table 4 that the PER is around 3\%-6\% which is comparable to typical PERs observed in traditional multiple access wireless communication systems. We estimate the theoretical throughput considering a PER range of $3 \%$ to $6 \%$, and error control code rate of $1 / 2$ as $0.94-0.97 \mathrm{Mbps}$, where Throughput $\approx(1-P E R) *$ packets $/ \mathrm{sec} *$ bits / packet $*$ errorcontrolcoderate. 
Table 4. Packet error rate (PER) calculated from received signal traces from each pixel OPEN duration of $T s=2$ s. Pixel 1(2) corresponds to signal from LED 1(2).

\begin{tabular}{cccc}
\hline \multirow{2}{*}{ Freq(Hz) } & \multirow{2}{*}{ \# Pkts in Ts } & \multicolumn{2}{c}{ Packet Error Rate (PER) (\%) } \\
\cline { 3 - 4 } & & Pixel 1 & Pixel 2 \\
\hline $500 \mathrm{kHz}$ & 477 & $5.88 \%$ & $5.46 \%$ \\
$1 \mathrm{MHz}$ & 954 & $4.83 \%$ & $2.63 \%$ \\
$2 \mathrm{MHz}$ & 1908 & $3.36 \%$ & $3.25 \%$ \\
\hline
\end{tabular}

\subsection{Impact of Switching Latency}

The switching latency is the effective time taken by the shutter receiver and its associated processing to switch the state and control from one pixel to another. Switching latency can impact the signal quality from each pixel during the shutter control phases. The switching latency is a function of the per-pixel time slot duration $T_{S}$ and the intermediate state (open/close) switching time per pixel. We compute the switching latency $=\left[(\right.$ pixels - to - scan $) \times\left(T_{s}+\right.$ switching - time - per - pixel $\left.)\right]+$ $[($ no. - of - transmitters $\times$ packet - size $\times$ switching - time - per - pixel $)]$, where the first part of the sum is the Step 1 latency and second part corresponds to Step 2. We first validated the consistency of the SNR values under different time slot duration selections for the pixels in our current prototype. Considering LED 1 as desired signal and with LED 2 switched OFF, we alternated pixels 1 and 2 to be OPEN for the specific time slot duration and recorded signal and noise power. We report the average SNR values in Table 5 and observe the consistency of SNR for short $(100 \mathrm{~ms})$ as well as long time slot durations ( $2 \mathrm{~s})$. The experiments were conducted in a well lit (white ceiling lighting) lab environment. The ambient light from the ceiling light is considered as the noise source, with a recorded average voltage was $4.9 \mathrm{mV}$ across all experiments; to help compare, the LED signal from $16 \mathrm{~cm}$ recorded about $0.6 \mathrm{~V}$ on the photodiode without any shutter.

Table 5. Average Signal to Noise Ratio (SNR) in $\mathrm{dB}$ for each pixel OPEN under different shutter switching times. Here, the LED transmitter is placed such that it illuminates Pixel 1 only.

\begin{tabular}{cccc}
\hline Pixels & Pixel 1 & Pixel 2 & $\boldsymbol{T}_{\boldsymbol{s}}[\mathrm{ms}]$ \\
\hline \multirow{4}{*}{$S N R_{d b}$} & 19.97 & -0.27 & $100 \mathrm{~ms}$ \\
& 19.96 & 0.48 & $500 \mathrm{~ms}$ \\
& 19.86 & -0.47 & $1000 \mathrm{~ms}$ \\
& 19.99 & -0.60 & $1500 \mathrm{~ms}$ \\
\hline
\end{tabular}

In our prototype the time slot duration is $100 \mathrm{~ms}$, pixel switching time is $1 \mathrm{~ms}$, and there are 2 transmitters sending 2096 bits packets. We measure the Step 1 (discovery) phase switching latency to be about $400 \mathrm{~ms}(2 \times(100 \mathrm{~ms}+1 \mathrm{~ms}))$ with a $5 \mathrm{~s}$ periodicity, and Step 2 (identification) phase switching latency to be about $4.2 \mathrm{~s}(2 \times 2096 \times 1 \mathrm{~ms})$. Ideally, the smallest shutter pixel switching time is desired, however, the hardware choice may cause a constraint. We observe that our LCD shutters in our prototype can go lowest up to $1 \mathrm{~ms}$ operation. If we were to consider practical usage of switching times of $T_{s}=1 \mathrm{~ms}$ (potentially using Digital Micromirror Devices DMDs), and shutter resolutions of $100 \times 100$ and $1000 \times 1000$ pixels, and even considering an overestimated number of 100 effective transmitters (which map to 100 different pixels) the effective, theoretically estimated, processing time (latency) of Steps 1 and 2 in Algorithm 1 would be about $220 \mathrm{~ms}$ and $1.2 \mathrm{~s}$, respectively. Such latency numbers can be considered practical for typical VLC applications including sensing, IoT and low-speed device-device data transfers. 


\section{Extended Evaluation: Mobile Scenario}

We extend the evaluation of our prototype system across mobile environments. The mobility considerations in these evaluations refer to the the case when the VLC transmitter can potentially change its spatial position while transmitting data. The receiver is kept stationary in all these experiments. When an actively transmitting LED changes its position, the receiver must be able to actively identify that the movement has happened and that it needs to adapt its reception area. Using our pixelated shutter approach, we hypothesize that when the LED changes position, the receiver will identify the movement event based on its periodic SNR measurements across each pixel and the automated shutter control protocol (Algorithm 1) shifts control to the appropriate pixel over which the LED signal is now being received. The goal of these evaluations is to verify the feasibility of our system to adapt its reception when the LED changes position.

\subsection{Experiment Setup and Methodology}

To facilitate controlled movements of the LED transmitter across different positions, we set up our VLC system on a table top with the receiver in static position. We 3D printed a housing for each of the two LED transmitters to be integrated with a 3-wheel RaspberryPi controlled robot as shown in Figure 5. With a distance of $0.5 \mathrm{~m}$ set between the VLC transmitter and receiver, we consider three key positions of the transmitter; A, B and OFOV (out of Field-of-View):

1. Position $\mathrm{A}$ is the point from where the LED transmitter illuminates Pixel 1 area of the shutter. When the robot is in position $\mathrm{A}$, the receiver must be able to identify the signal is on Pixel 1 and only OPEN Pixel 1 (keeping others closed).

2. Position B is the point from where the LED transmitter illuminates Pixel 2 area of the shutter. When the robot is in position $B$, the receiver must be able to identify the signal is on Pixel 2 and only OPEN Pixel 2 (keeping others closed).

3. Out of Field of Views (OFOV) are the marked positions on the straight line trajectory of the robot where the LED signals are either minimal or out of field-of-view of the receiver lens and photodiode. When the robot is in this position, the receiver must identify there is no active transmission from the LED being received and hence must close all pixels to avoid any noise or interference signals being sampled on the receiver.

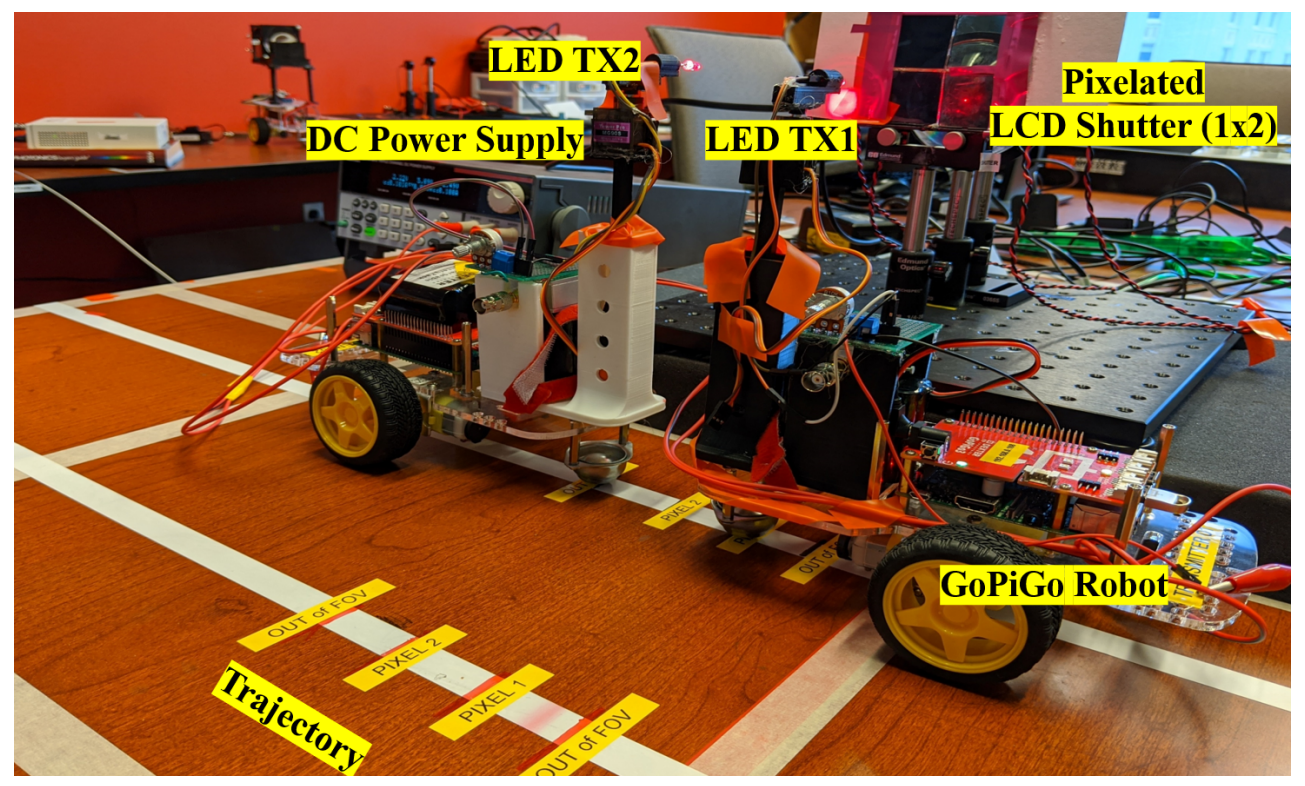

Figure 5. LED transmitters placed on GoPiGo RaspberryPi robots. 


\subsection{Results}

We set the robot to move from one end of the table to the other along a straight line trajectory, traversing positions A, B and two marked out-of-FOV points (see Figure 6 for an illustration). In each experiment trial we chose a specific speed of the robot and shutter switching time and move the robot along the calibrated trajectory. The receiver selects the pixel status as OPEN/CLOSE based on, first computing the SNR on each pixel and flagging the pixels which have SNR above an empirical threshold (Table 5) for each switching time selection, and then the receiver making a recommendation on which pixels to OPEN/CLOSE based on whether it has detected the ID sequence (decode bits, correlate with sequence and flag success if correlation above 90\%). We set our experimentation to record the system's pixel shutter recommendations in the form of a 2 bit binary representation as listed in Table 6. For example, a 01 implies that the system correctly identified that Pixel 1 should be open and identified the signal on Pixel 1 using the 5 bit Barker ID sequence transmitted as payload.
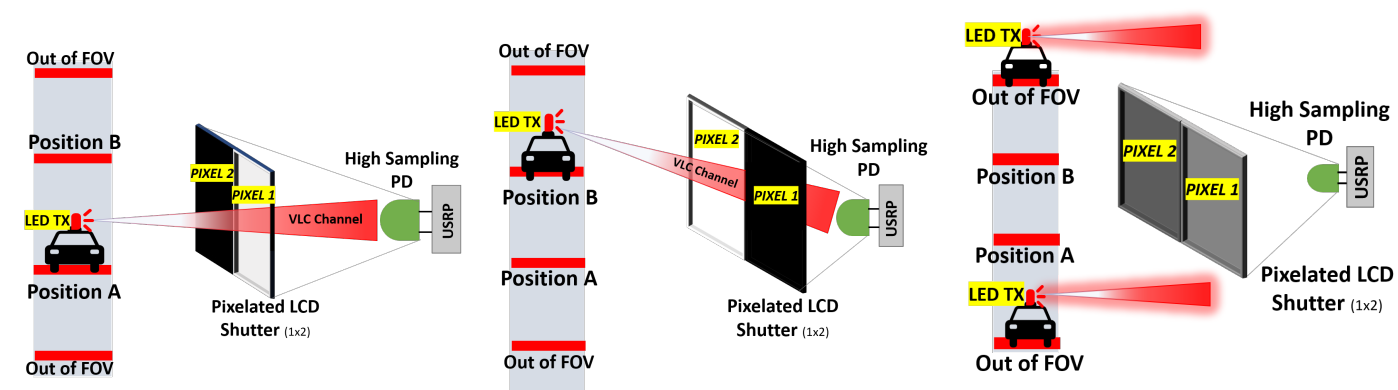

Figure 6. Mobility experiments with the variation of LED Transmitter's position: A (left), B (center) and Out of Field of Views (OFOV) (right).

Table 6. Binary mapping of the output or shutter pixels status recommendations.

\begin{tabular}{cc}
\hline Configurations & Binary Representation \\
\hline Pixel 2 Closed, Pixel 1 Closed & 00 \\
Pixel 2 Closed, Pixel 1 Open & 01 \\
Pixel 2 Open, Pixel 1 Closed & 10 \\
Pixel 2 Open, Pixel 1 Open & 11 \\
\hline
\end{tabular}

In Figure 7, we report our system recommendation output for three speeds of the robot (20 dps or $0.53 \mathrm{~cm} / \mathrm{s}, 15 \mathrm{dps}$ or $0.4 \mathrm{~cm} / \mathrm{s}, 10 \mathrm{dps}$ or $0.26 \mathrm{~cm} / \mathrm{s}$ ) and $1000 \mathrm{~ms}$ shutter switching time. There is an ERROR case of recommendation where both pixels are open (binary representation: 11) in both 15 and $10 \mathrm{dps}$ robot's speed experimentation. During this time, the robot's position is at the intersection of A \& B, where both pixels are illuminated at the same time and the measured SNR values in both pixels are higher than the threshold value. However, we observed over 5 trials, even in the reported least accurate case, the ERROR cases occur largely in the transition phase from one pixel to another. This can be resolved by synchronizing the transition time with the speed of the robot or by speeding up shutter sampling (smaller intervals) so that even if the error occurs during a transition, there are enough samples to identify the behavior and a suitable action can be taken on the receiver. Further, more receiver samples imply possibility of using probabilistic estimation of whether the LED has changed position or not. 


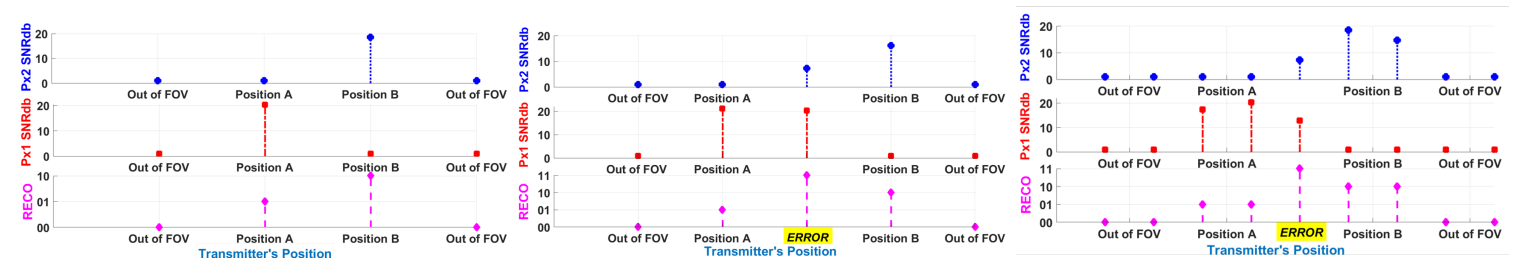

Figure 7. Our system's output (recommendations) at $1 \mathrm{~s}$ shutter switching time and at different speeds of the robot: $20 \mathrm{dps}$ (left), $15 \mathrm{dps}$ (center), $10 \mathrm{dps}$ (right). We conducted 5 trials and ordered the highest to least accuracy. These figures represent the least accuracy case.

As an extended experiment we evaluate how the system performs for much smaller sampling time intervals such as $100 \mathrm{~ms}$ and $50 \mathrm{~ms}$. These sampling intervals can cater to real-time human-computer interaction applications and other applications using real-time feedback. While the focus of this work is not necessarily to emphasize only one category of application, we do explore on the success and limitations of our system under such fast sampling time settings. We report the results from our robot movement based experiment results, discussed previously, for these cases and report them in Figures 8 and 9, respectively. We observe that in most sampled points the system recommends correctly, however, some cases of error when the LEDs are not in FOV or transitioning. These errors can be resolved by employing statistical filtering to the recommendation/identification results in each sample over the duration of a reasonable and practical time window. For example, even if the sampling duration is $100 \mathrm{~ms}$, the samples across a $1 \mathrm{~s}$ time window can be considered for statistical estimation and filtering noise to ensure the correct receiver sampling area is accounted. Another issue, the leakage of LED signals through the shutter even if it is CLOSED. We note that we have used an off-the-shelf LCD shutter, which has only a $95 \%$ marked opacity, which means that $5 \%$ of the ambient and incoming light on the shutter are still let through. At these short distances we believe the leakage is causing significant changes in the SNR values and hence leading to erroneous recommendations when both pixels are CLOSED or both OPEN. One option is to replace the LCD shutter with Digital Micromirror Device (DMD) based high speed shutters to ensure much faster and efficient pixel switching. In summary, we note that the goal of our current work is to explore these mobile scenarios and make observations of the artifacts arising in our system response. We reserve the optimization of the system performance for better hardware and across mobile scenarios for future work.

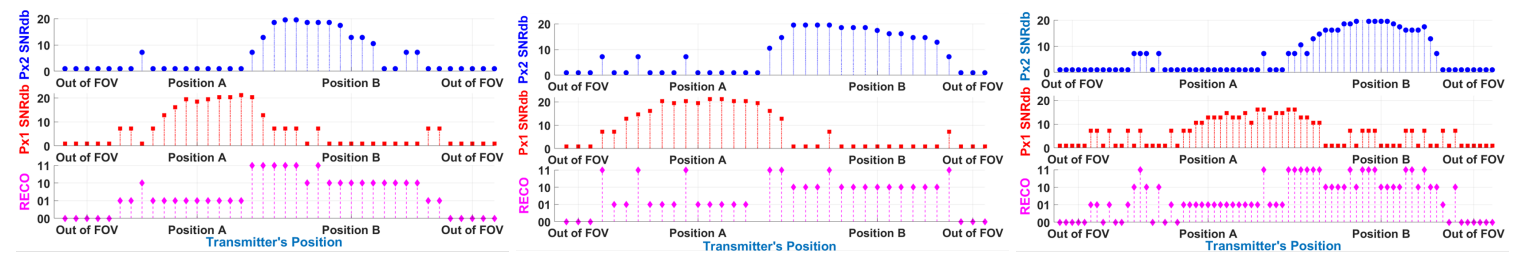

Figure 8. Our system's output (recommendations) at $100 \mathrm{~ms}$ shutter switching time and at different speeds of the robot: $20 \mathrm{dps}$ (left), $15 \mathrm{dps}$ (center), $10 \mathrm{dps}$ (right).

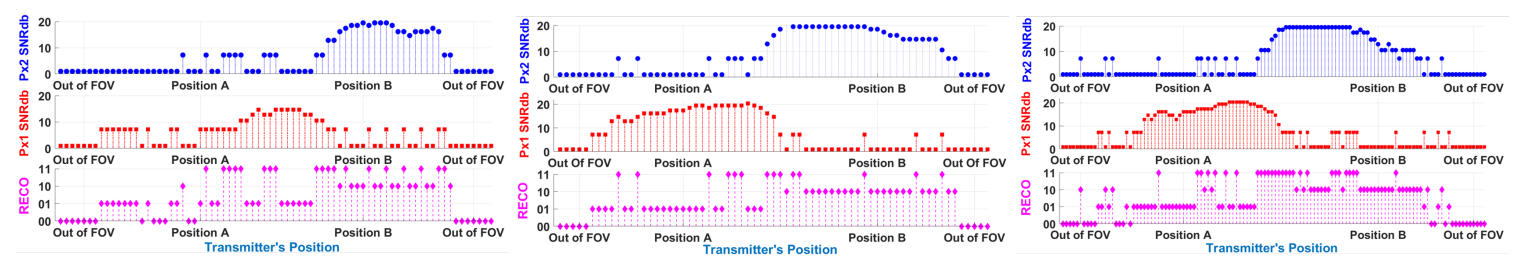

Figure 9. Our system's output (recommendations) at $50 \mathrm{~ms}$ shutter switching time and at different speeds of the robot: $20 \mathrm{dps}$ (left), $15 \mathrm{dps}$ (center), $10 \mathrm{dps}$ (right).

\section{Discussion}

Ideally, the size of the LCD pixel area has to be matched with the size of the LED signal blob on the receiver. The size of the blob changes (decreases) with (increasing) distance between the transmitter 
and receiver. It will be appropriate to have an extremely small LCD pixel area to ensure the signal from the maximum range of the LED such that the signal on the photodiode is above ambient noise (usually meters to $10 \mathrm{~s}$ of meters). In that regard, we originally chose to use a TFT LCD pixel array of a small TFT LCD monitor screen (used for Arduinos and Raspberry Pis). However, due to the hardware and software limitations in controlling separate pixels, we chose to build a contraption of an LCD pixel array and the ones we have used are the smallest size that can be obtained off-the-shelf. In our next iteration of this design we plan to use Digital Micromirror Devices (DMD) to achieve small areas of reception as well as faster switching times.

\section{Conclusions and Future Work}

In this paper, we introduced a novel architecture and a protocol to enable multiple access reception on a VLC receiver with only a single photodiode element. We designed and evaluated a system that enables transmission from 2 LEDs simultaneously and selectively decodes packets from each based on a selection algorithm that uses OPEN/CLOSE cycles of LCD shutter pixels acting as digital apertures for the photodiode signal. Through BER, PER and latency metrics (processing latency), computed through experiments, we showed the feasibility and performance of our 2 transmitter-to- 1 receiver multiple access system at low and high signal frequencies. We also successfully verified the feasibility of the use of the system across controlled mobile settings by setting the LED transmitters on robots and the pixelated receiver being static. To the best of our knowledge, this work sets the foundation stage for future work in multiple access using single photodiode receiver.

Author Contributions: Project administration, methodology, software, validation, writing-original draft preparation, M.R.R.; hardware, experimentation, writing contribution, K.A.; conceptualization, writing-review and editing, supervision, funding acquisition, A.A. All authors have read and agreed to the published version of the manuscript.

Funding: This work has been supported by the CISE CRII Program of the National Science Foundation under the grant NSF CRII CNS-NeTS 1755925 and REU Supplement grant CNS-1929171.

Acknowledgments: The authors would like to thank all the reviewers for their valuable comments to help polish this paper. The authors would also like to extend their thanks to Yashaswini Vishwanath, a M.S. in CS candidate at Georgia State University for extending help in setting up the robot for experiments. The authors would like to thanks the National Science Foundation (NSF) for funding this research, and the Department of Computer Science at Georgia State University for supporting this research.

Conflicts of Interest: The authors declare no conflict of interest.

\section{References}

1. Kahn, J.; Barry, J. Wireless infrared communications. Proc. IEEE 1997, 85, 265-298. [CrossRef]

2. Komine, T.; Nakagawa, M. Fundamental analysis for visible-light communication system using LED lights. IEEE Trans. Consum. Electron. 2004, 50, 100-107. [CrossRef]

3. Ashok, A. Design, Modeling and Analysis of Visual MIMO Communication. Ph.D. Thesis, Rutgers University, New Brunswick, NJ, USA, 2014.

4. Rajbhandari, S.; Jalajakumari, A.V.N.; Chun, H.; Faulkner, G.; Cameron, K.; Tsonev, D.; Haas, H.; Xie, E.; McKendry, J.J.D.; Herrnsdorf, J.J.D.; et al. A Multi-Gigabit/sec Integrated Multiple Input Multiple Output VLC Demonstrator. J. Lightwave Technol. 2017. [CrossRef]

5. Rahman, M.R.; Islam, S.M.T.; Ashok, A. A Novel Architecture for Ultra-High Signal-to-Interference-NoiseRatio Reception in Visible Light Communication. In Proceedings of the 2019 11th International Conference on Communication Systems Networks (COMSNETS), Bangalore, India, 7 January 2019; pp. 172-179. [CrossRef]

6. pureLiFi: The LiFi Technology. Available online: http:/ / purelifi.com/ (accessed on 10 May 2020).

7. Galisteo, A.; Juara, D.; Giustiniano, D. Research in Visible Light Communication Systems with OpenVLC1.3. In Proceedings of the 2019 IEEE 5th World Forum on Internet of Things (WF-IoT), Limerick, Ireland, 18 April 2019; pp. 539-544. [CrossRef] 
8. Haus, M.; Ding, A.Y.; Ott, J. LocalVLC: Augmenting Smart IoT Services with Practical Visible Light Communication. In Proceedings of the 2019 IEEE 20th International Symposium on "A World of Wireless, Mobile and Multimedia Networks" (WoWMoM), Washington, DC, USA, 10 June 2019; pp. 1-9. [CrossRef]

9. Yin, S.; Smaoui, N.; Heydariaan, M.; Gnawali, O. Purple VLC: Accelerating Visible Light Communication in Room-Area through PRU Offloading. In Proceedings of the International Conference on Embedded Wireless Systems and Networks (EWSN) 2018, Madrid, Spain, 14-16 February; pp. 67-78.

10. Liu, C.; Jin, X.; Zhu, W.; Jin, M.; Xu, Z. Demonstration of a low complexity ARM-based indoor VLC transceiver under strong interference. In Proceedings of the 2017 13th International Wireless Communications and Mobile Computing Conference (IWCMC), Valencia, Spain, 28 June 2017; pp. 622-627. [CrossRef]

11. Yin, S.; Gnawali, O. Towards Embedded Visible Light Communication Robust to Dynamic Ambient Light. In Proceedings of the 2016 IEEE Global Communications Conference (GLOBECOM), Washington, DC, USA, 8 December 2016; pp. 1-6. [CrossRef]

12. Marshoud, H.; Kapinas, V.M.; Karagiannidis, G.K.; Muhaidat, S. Non-Orthogonal Multiple Access for Visible Light Communications. IEEE Photonics Technol. Lett. 2016, 28, 51-54. [CrossRef]

13. Chen, C.; Zhong, W.; Yang, H.; Du, P. On the Performance of MIMO-NOMA-Based Visible Light Communication Systems. IEEE Photonics Technol. Lett. 2018, 30, 307-310. [CrossRef]

14. Eltokhey, M.W.; Khalighi, M.; Ghassemlooy, Z. Multiple Access Techniques for VLC in Large Space Indoor Scenarios: A Comparative Study. In Proceedings of the 2019 15th International Conference on Telecommunications (ConTEL), Graz, Austria, 3-5 July 2019; pp. 1-6. [CrossRef]

15. Pefkianakis, I.; Hu, Y.; Wong, S.H.; Yang, H.; Lu, S. MIMO rate adaptation in 802.11n wireless networks. In Proceedings of the Sixteenth Annual International Conference on Mobile Computing and Networking, Chicago, IL, USA, 20 September 2010; pp. 257-268. [CrossRef]

16. Khan, S.M.; Saha, N.; Rahman, M.R.; Hasan, M.; Rahman, M.S. Performance improvement of MIMO VLC using V-BLAST technique. In Proceedings of the 2016 th International Conference on Informatics, Electronics and Vision (ICIEV), Dhaka, Bangladesh, 14 May 2016; pp. 45-49. [CrossRef]

17. Azhar, A.H.; Tran, T.; O’Brien, D. A Gigabit/s Indoor Wireless Transmission Using MIMO-OFDM Visible-Light Communications. IEEE Photonics Technol. Lett. 2013, 25, 171-174. [CrossRef]

18. Zhu, Y.; Liang, W.; Zhang, J.; Zhang, Y. Space-Collaborative Constellation Designs for MIMO Indoor Visible Light Communications. IEEE Photonics Technol. Lett. 2015, 27, 1667-1670. [CrossRef]

19. Ijaz, M.; Tsonev, D.; McKendry, J.J.D.; Xie, E.; Rajbhandari, S.; Chun, H.; Faulkner, G.; Gu, E.; Dawson, M.D.; O'Brien, D.; et al. Experimental proof-of-concept of optical spatial modulation OFDM using micro LEDs. In Proceedings of the 2015 IEEE International Conference on Communication Workshop (ICCW), London, UK, 12 June 2015; pp. 1338-1343.

20. Lian, J.; Brandt-Pearce, M. Multiuser MIMO Indoor Visible Light Communication System Using Spatial Multiplexing. J. Lightwave Technol. 2017, 35, 5024-5033. [CrossRef]

21. Kobayashi, K.; Kobayashi, H.; Iwashita, K. Multi-User MIMO in Visible Light Communications by Superposed Intensity Modulated Signals. In Proceedings of the 2019 24th OptoElectronics and Communications Conference (OECC) and 2019 International Conference on Photonics in Switching and Computing (PSC), Fukuoka, Japan, 11 July 2019; pp. 1-3. [CrossRef]

22. Xiao, Y.; Zhu, Y.; Zhang, D.; Zhang, H. High-Speed Visible Light Communication Chipset Based Multi-Color MIMO System. In Proceedings of the 2019 28th Wireless and Optical Communications Conference (WOCC), Beijing, China, 9 May 2019; pp. 1-5. [CrossRef]

23. Pham, T.V.; Minh, H.L.; Pham, A.T. Multi-cell VLC: Multi-user downlink capacity with coordinated precoding. In Proceedings of the 2017 IEEE International Conference on Communications Workshops (ICC Workshops), Paris, France, 29 May 2017; pp. 469-474. [CrossRef]

24. Grobe, L.; Paraskevopoulos, A.; Hilt, J.; Schulz, D.; Lassak, F.; Hartlieb, F.; Kottke, C.; Jungnickel, V.; Langer, K. High-speed visible light communication systems. IEEE Commun. Mag. 2013, 51, 60-66. [CrossRef]

25. Chen, S.; Chow, C. Color-Shift Keying and Code-Division Multiple-Access Transmission for RGB-LED Visible Light Communications Using Mobile Phone Camera. IEEE Photonics J. 2014, 6, 1-6. [CrossRef]

26. Chun, H.; Gomez, A.; Quintana, C.; Zhang, W.; Faulkner, G.; O’Brien, D. A Wide-Area Coverage 35 Gb/s Visible Light Communications Link for Indoor Wireless Applications. Sci. Rep. 2019, 9, 1-8. [CrossRef] [PubMed] 
27. Max Born, E.W. Principles of Optics; Cambridge University Press: Cambridge, UK, 1999.

28. Jang, Y.M.; Aoyama, H.; Roberts, R.; Zeng, Y.; Oshima, M.; Chitrakar, R.; Serafimovski, N.; Uysal, M.; Cha, J.; Chang, S.; et al. IEEE 802.15 TG7 Technical Considerations Document; IEEE: Piscataway, NJ, USA, 2015.

(C) 2020 by the authors. Licensee MDPI, Basel, Switzerland. This article is an open access article distributed under the terms and conditions of the Creative Commons Attribution (CC BY) license (http://creativecommons.org/licenses/by/4.0/). 